Results 60 patients underwent colonoscopy for colonic tumors. The average age was 55.60 years old, $53.3 \%$ were females, $85 \%$ had a family history of colon cancer and 50\% were smokers. A significant association of Pap stain in detecting malignancy $(p=0.002)$ was noted with a sensitivity of $79.2 \%$ and specificity of $66.6 \%$.

Conclusions The results show good sensitivity of using Pap stain in that it is able to correctly identify $79.2 \%$ for patients who truly have colonic tumors with specificity of $66.6 \%$. There is a significant association between Pap stain and H\&E stain in detecting malignancy $(p=0.002)$ in that a positive Pap stain is highly associated with colon cancer detection $(\mathrm{PPV}=90.5 \%)$ and only $44.4 \%$ will be truly negative for those who have no colon cancer.

\section{IDDF2019-ABS-0324 APPROPRIATENESS OF PRESCRIBING PROTON PUMP INHIBITOR IN A TERTIARY CARE SUPERSPECIALITY HOSPITAL IN INDIA}

Nagesh Kamat*, Shiran Shetty, Sumit Bhatia, Anurag Shetty. Department of Gastroenterology and Hepatology, Kasturba Medical College, Manipal Academy of Higher Education, Manipal, India

\subsection{6/gutjnl-2019-IDDFabstracts.232}

Background Proton Pump Inhibitor (PPI) is the commonest prescribed drug in gastroenterology practice due to its proven effectiveness for a variety of indications. Long-term use contributes to adverse events. There is a large unmet need to evaluate the appropriateness of prescribing PPI therapy.

Methods A cross-sectional study was conducted over three months at a Gastroenterology outpatient department of a tertiary care teaching hospital. Adult patients who were prescribed PPI were interviewed. Patient medication charts were reviewed and demographic details, PPI use, dose, regimen and valid indication for PPI prescription was noted in accordance with international guidelines and best evidence.

Results Three forty-one (male 240; 70.4\%) patients were included. Their median age was 35 (range 18-78) years. $191(56 \%), 84(24.6 \%)$ and $56(16.4 \%)$ of the patients received oral Rabeprazole, Pantoprazole and Esomeprazole respectively. Rest were prescribed a combination of PPI with prokinetic (Domperidone/Itopride). The commonest indication was Gastroesophageal Reflux Disease followed by Pancreatitis. Overall, $10(2.9 \%)$ of the patients were receiving PPIs without an appropriate indication out of which $\underline{3}$ were on self-medication. $85(24.9 \%)$ patients had a previous PPI prescription, and 256(75.1\%) were initiated on PPI therapy during their current visit. The median duration of therapy was 4.5 weeks.

Conclusions Majority of the patients (97.1\%) had an identifiable and appropriate PPI indication. Accurate PPI prescribing can reduce the incidence of adverse events and contribute to overall cost savings. Nevertheless, patients should be educated and warned regarding self-medication and inappropriate use. Meanwhile, doctors need to reevaluate drugs at follow up, reduce dose and stop accordingly.

\section{IDDF2019-ABS-0328 THE USE OF HEMOSPRAY IN UPPER GI BLEEDING: A SINGLE CENTER EXPERIENCE IN A DEVELOPING COUNTRY}

Leila Sawadjaan*, Josef Carlo Lazaro. The Medical City Section of Gastroenterology, Philippines

\subsection{6/gutjnl-2019-IDDFabstracts.233}

Background Hemospray and other endoscopic hemostatic sprays are relatively new techniques for upper GI bleeding. Due to its cost, physicians are wary of its use and efficacy. Hemostasis in upper gastrointestinal bleeding can be obtained depending on the type of lesion and extent of bleeding through the following Methods injection of saline diluted epinephrine solution, the application of various types of clips, or using argon plasma- coagulation and other thermic coagulation procedures. Despite these available techniques, about $30 \%$ of patients with upper GI bleeding cannot achieve hemostasis endoscopically. Hemospray is the only available sprayable powder in the Philippines so far, and it is being considered as an alternative method. Hence this study aimed to report on the experience of a single tertiary center in the Philippines on the use of Hemospray in upper GI bleeding.

Methods The endoscopy database of The Medical City Hospital was reviewed retrospectively from July 2017 to February 2019 and and eight cases were included in this study. The main clinical presentation of the eight patients was overt upper GI bleeding, presenting as gross hematemesis and melena, and were treated with Hemospray (Cook Medical). Bleeding was secondary to gastric cancer (2), gastric polyp (3), esophageal varix (2) and metastates to stomach (1) that were all unresolved by conventional therapeutic gastroscopy manouvres including epinephrine injection, rubber band ligation, and thermic coagulation. (figure 1)

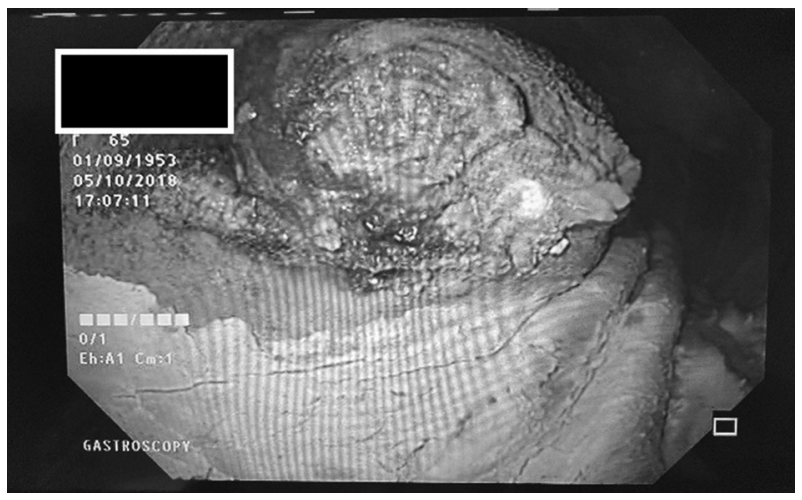

Abstract IDDF2019-ABS-0328 Figure 1

Results In all of the cases presented, hemostasis was achieved abruptly by use of Hemospray. Average time until cessation of bleeding was less than one minute. Among the successful cases, two re-bled after a certain time after hemostasis. Average time until recurrence of bleeding was 36 hours. The average length of hospital stay was 14.5 days. No complications related to Hemospray occurred within the next 48 hours postprocedure.

Conclusions Hemospray may be considered as an effective and safe method for the endoscopic management of acute upper 